Supporting Information

\title{
Universal Fluorination-Created Edge C-F Groups in Networks of Multidimensional Carbon Materials
}

Yongjie $\mathrm{Xu}^{\dagger}$, Shuilin $\mathrm{Li}^{\dagger}$, Weili Zhang ${ }^{\dagger}$, Yuan Liu ${ }^{\dagger, \ddagger}$, Jiawei $\mathrm{Liu}^{\dagger}$, Maoyun $\mathrm{Di}^{\dagger}$, Yong Wang ${ }^{\dagger, \S}$, Youwei Du, ${ }^{\dagger}$ and Nujiang Tang ${ }^{*}$,

${ }^{\dagger}$ National Laboratory of Solid State Microstructures, Collaborative Innovation Center of Advanced Microstructures, and Jiangsu Provincial Key Laboratory for Nanotechnology, Nanjing University, Nanjing 210093, China, 抽aculty of Science, Zhenjiang Key Laboratory for Advanced Sensing Materials and Devices, Jiangsu University, Zhenjiang 212013, China, and §Academy of Advanced Interdisciplinary Research, Xidian University, Xi'an, 710126, China

Email address: tangnujiang@nju.edu.cn

\section{Experimental Methods}

Graphene (viz. $\mathbf{G}_{\mathrm{Ar}}$ ). Graphene was synthesized by annealing of graphene oxide (GO) in Ar at $700{ }^{\circ} \mathrm{C}$ for $1 \mathrm{~h}$. Herein, GO was prepared by improved Hummers' method. ${ }^{1}$ In a typical experiment, the mixture of $3 \mathrm{~g}$ graphite, $360 \mathrm{ml}$ condensed $\mathrm{H}_{2} \mathrm{SO}_{4}$, and $40 \mathrm{ml}$ condensed $\mathrm{H}_{2} \mathrm{PO}_{4}$ was stirred for $1.5 \mathrm{~h}$ at $0{ }^{\circ} \mathrm{C}$ and added $18 \mathrm{~g} \mathrm{KMnO} 4$ and then followed by another $12 \mathrm{~h}$ stirring at $50{ }^{\circ} \mathrm{C}$. Thereafter, $400 \mathrm{ml} \mathrm{H}_{2} \mathrm{O}$ was added into the mixture within 15 min at a steady flow, and then $40 \mathrm{ml} \mathrm{H}_{2} \mathrm{O}_{2}$ was added within 6 min at a steady flow. The obtained solution was first washed with hydrochloric acid 10 times and then with deionized (DI) water 10 times. Thereafter, GO 
powder was obtained after freeze drying. Graphene was synthesized by annealing of GO in Ar at $700{ }^{\circ} \mathrm{C}$ for $1 \mathrm{~h}$.

GH2. GH2 was synthesized by annealing of GO in $\mathrm{H}_{2}$ at $450{ }^{\circ} \mathrm{C}$ for $2 \mathrm{~h}$ followed by $\mathrm{Ar}$ at $900{ }^{\circ} \mathrm{C}$ for 1 h. $^{2}$

SWCNTs. SWCNTs (XFS02, purity: > 90\%, outer diameter: $<2 \mathrm{~nm}$, length: 5-30 $\mu \mathrm{m}$ ) were purchased from Nanjing XFNANO Materials Tech Co., Ltd (Nanjing, China).

Fullerene. Fullerene (C60, purity: > 99\%) were purchased from Alfa Aesar A Johnson Matthey Company. Materials were ground for 30 minutes with alcohol as lubricant for preprocessing, in order to get more delicate and uniform C60.

FGi. FGi was bought from Shanghai CarFluor Chemicals Co., Ltd.

Fluorination of graphene. The FG samples under different pressures were obtained by annealing the mixture of graphene and $\mathrm{XeF}_{2}$ with different mass ratios in a Teflon container at $200{ }^{\circ} \mathrm{C}$ for $28 \mathrm{~h}$ in Ar. The FG samples under different temperatures were obtained by annealing the mixture of graphene and $\mathrm{XeF}_{2}$ with same mass ratio in a Teflon container at 100, 150 and 200 ${ }^{\circ} \mathrm{C}$ for $28 \mathrm{~h}$ in Ar, respectively. The $\mathrm{FG}_{\mathrm{Ar}}$ sample was prepared by annealing the mixture of $\mathrm{G}_{\mathrm{Ar}}$ and $\mathrm{XeF}_{2}$ in a Teflon container at $200{ }^{\circ} \mathrm{C}$ for $28 \mathrm{~h}$ in Ar.

Fluorination of $\mathbf{G H}_{2}$. The samples were obtained under different pressures (FG0.065, FG0.23, $\mathrm{FG}_{0.46}$ (viz. $\mathrm{FG}_{\mathrm{H} 2}$ ) and $\mathrm{FG}_{0.7}$, numeric numbers denote the pressure based on state equation during fluorination) by annealing the mixture of $\mathrm{GH}_{2}$ and $\mathrm{XeF}_{2}$ with different mass ratios in a Teflon container at $200{ }^{\circ} \mathrm{C}$ for $28 \mathrm{~h}$ in Ar. 
Fluorination of SWCNTs. The F-SWCNTs samples under different temperatures were obtained by annealing the mixture of SWCNTs and $\mathrm{XeF}_{2}$ with same mass ratio in a Teflon container at 200, 220 and $240{ }^{\circ} \mathrm{C}$ for $28 \mathrm{~h}$ in Ar respectively.

Fluorination of Fullerene. The F-C60 samples under different pressures were obtained by annealing the mixture of $\mathrm{C}_{60}$ and $\mathrm{XeF}_{2}$ with different mass ratios in a Teflon container at $200{ }^{\circ} \mathrm{C}$ for $28 \mathrm{~h}$ in Ar.

Microstructure characterization. The morphologies were investigated by transmission electron microscopy (TEM, FEI Tecnai-F20). X-ray photoelectron spectroscopy (XPS) measurements were carried on a PHI5000 VersaProbe (ULVAC-PHI, Japan) using $200 \mathrm{~W}$ mono-chromated Al Ka radiation. Raman spectra were obtained by confocal Raman microscope (LabRAM Aramis, Japan) using a laser excitation of $532 \mathrm{~nm}$. X-ray diffraction measurements were carried on Rigaku D/MAX-Ultima III using $\mathrm{Cu} \mathrm{K} \alpha$ radiation.

UV-vis measurements. The optical absorption spectra were recorded on a UV-vis spectrophotometer (Jasco V770) in the diffuse reflectance mode.

PL measurements. PL emission spectra (375 nm excitation) were measured at room temperature with a fluorescence spectrophotometer (Princeton Instruments, Acton SP2500).

Magnetic measurements. The magnetic properties of the powdered samples were measured using a superconducting quantum interference device (SQUID) magnetometer with a sensitivity better than $10^{-8}$ emu (Quantum Design MPMS-XL, USA), and all data were corrected for the diamagnetic contribution by subtracting the corresponding linear diamagnetic background 
measured at $300 \mathrm{~K}$. The concentrations of magnetic impurity elements (such as $\mathrm{Fe}$, Co, Ni or Mn) of all the samples are below 20 ppm (Supplementary Table 3) measured by ICP spectrometry (Jarrell-Ash, USA).

\section{Supporting figures and tables}
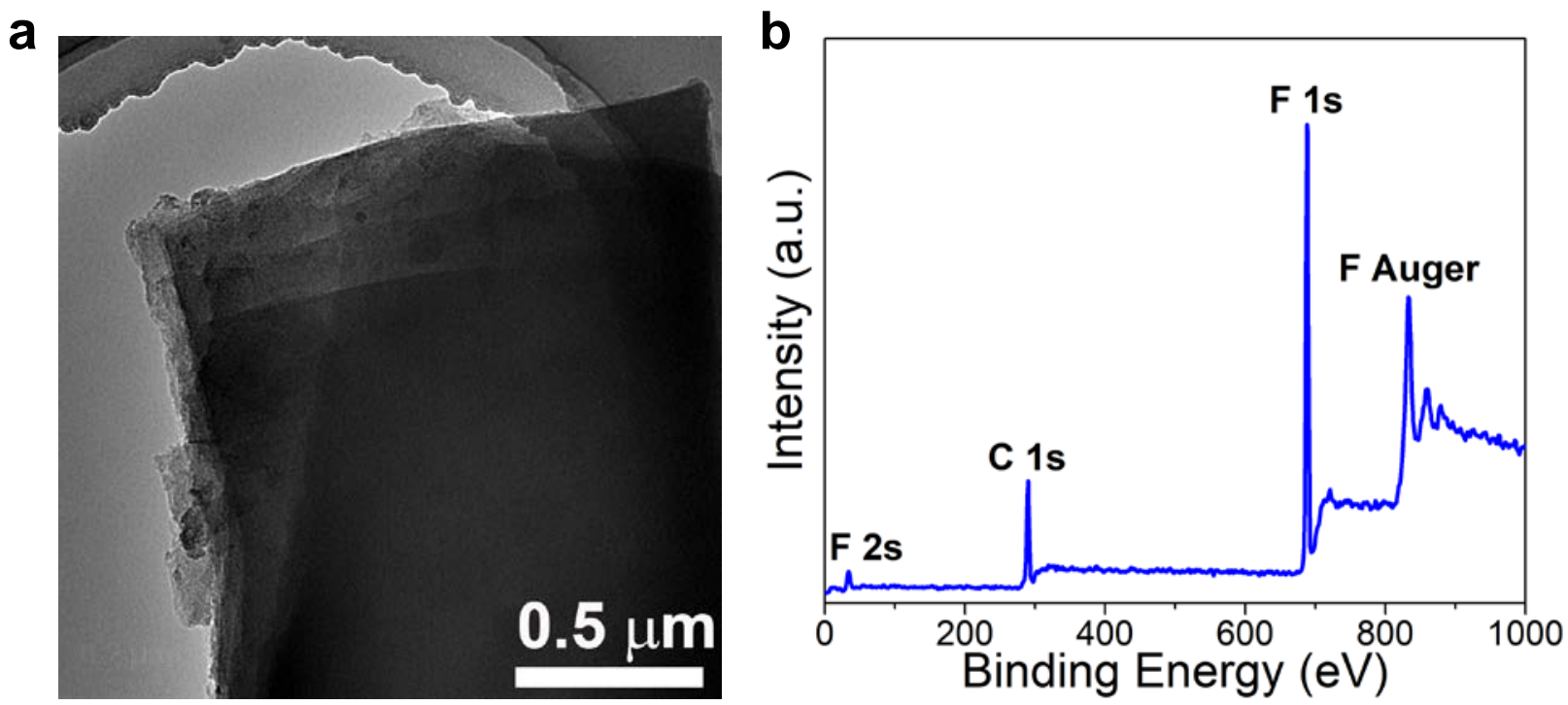

Figure S1. (a) TEM image. (b) XPS spectrum over a wide range of binding energies. FGi shows a typical bulk features with the micron-scale integrity. The fluorination degree is high up to 121.4 at\%. 
a

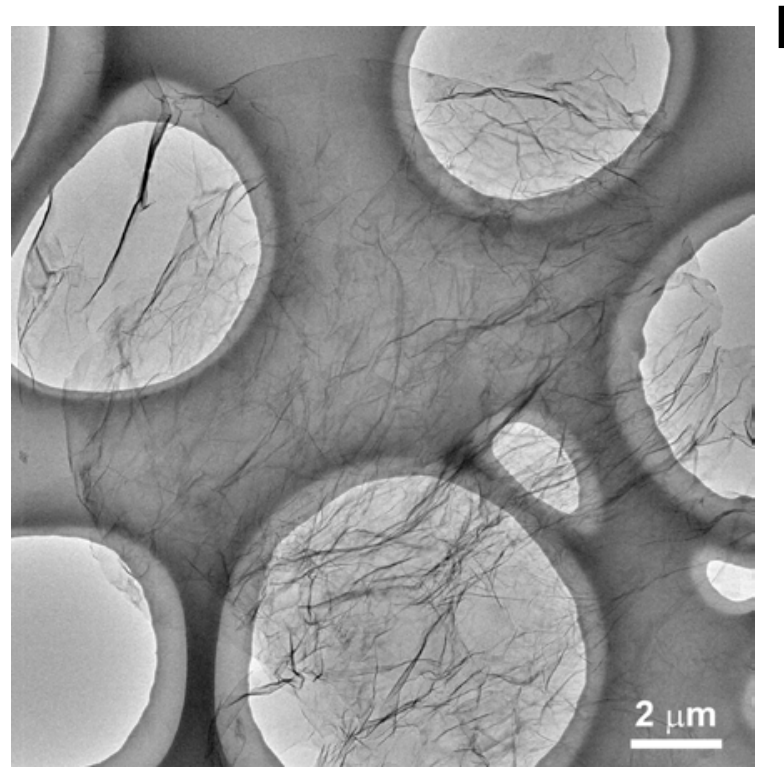

b

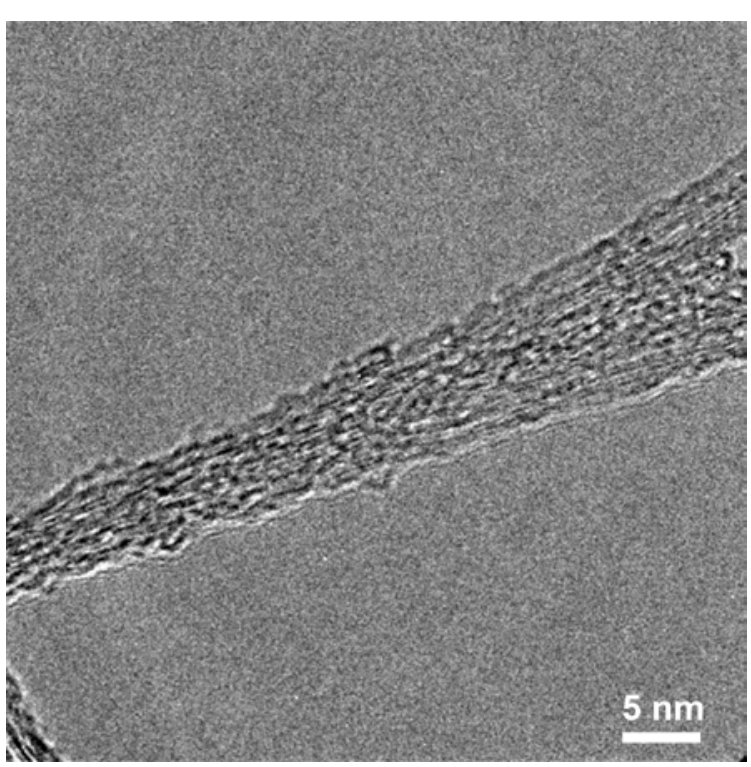

C

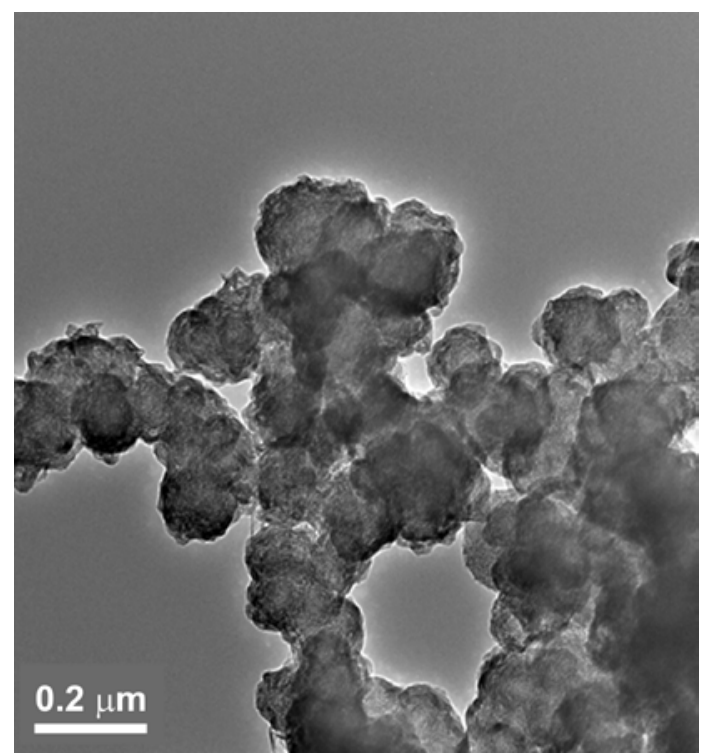

Figure S2. Typical TEM image of (a) graphene, (b) SWCNTs and (c) C60. Graphene shows a few sheets with the lateral size around $10 \mu \mathrm{m}$. The diameters of SWCNTs are 2-4 nm and the lateral sizes of $\mathrm{C}_{60}$ are mainly distributed in $50 \mathrm{~nm}$. 

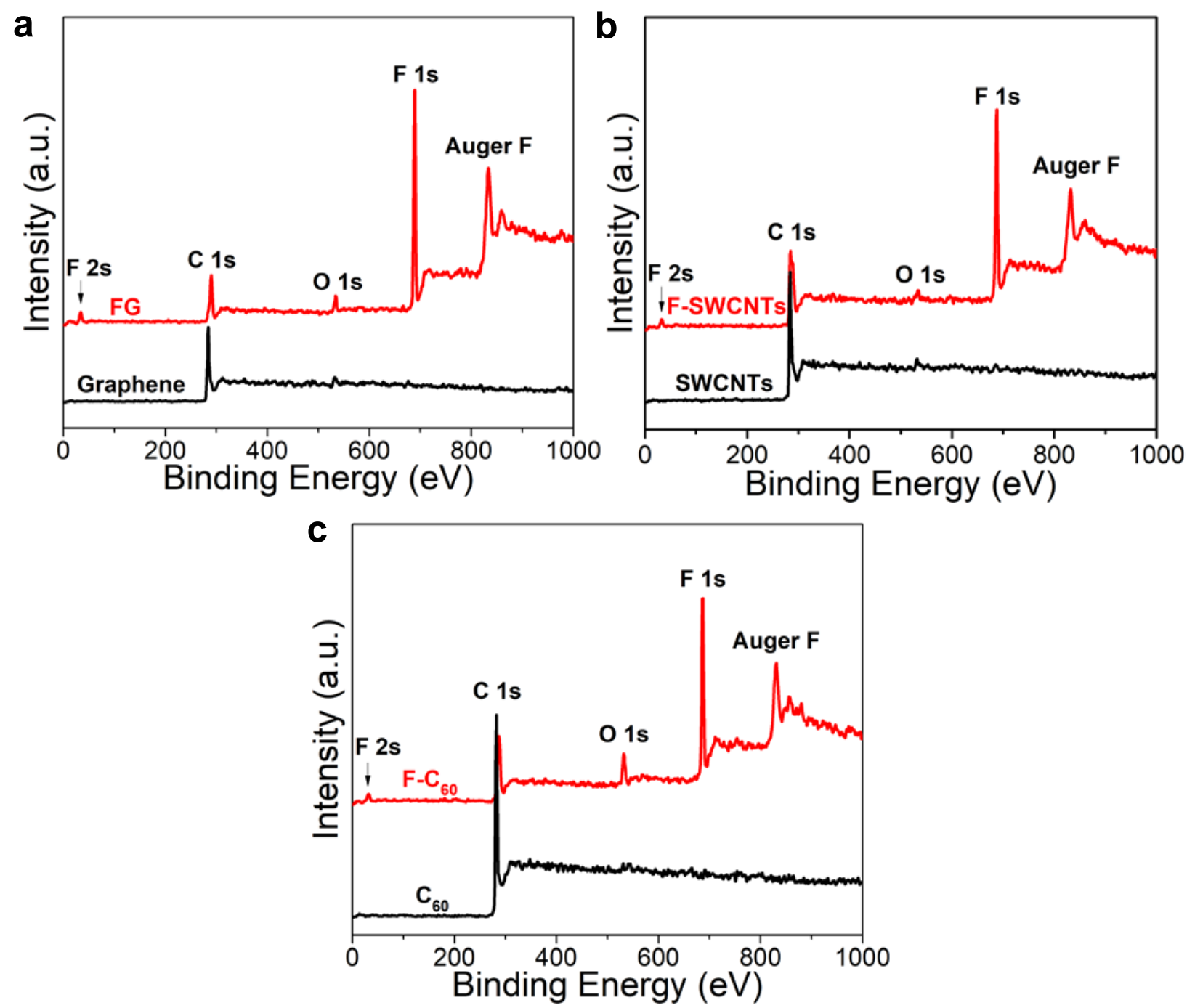

Figure S3. XPS spectra over a wide range of binding energies of (a) graphene and FG, (b) SWCNTs and F-SWCNTs, and (c) $\mathrm{C}_{60}$ and F-C60. 

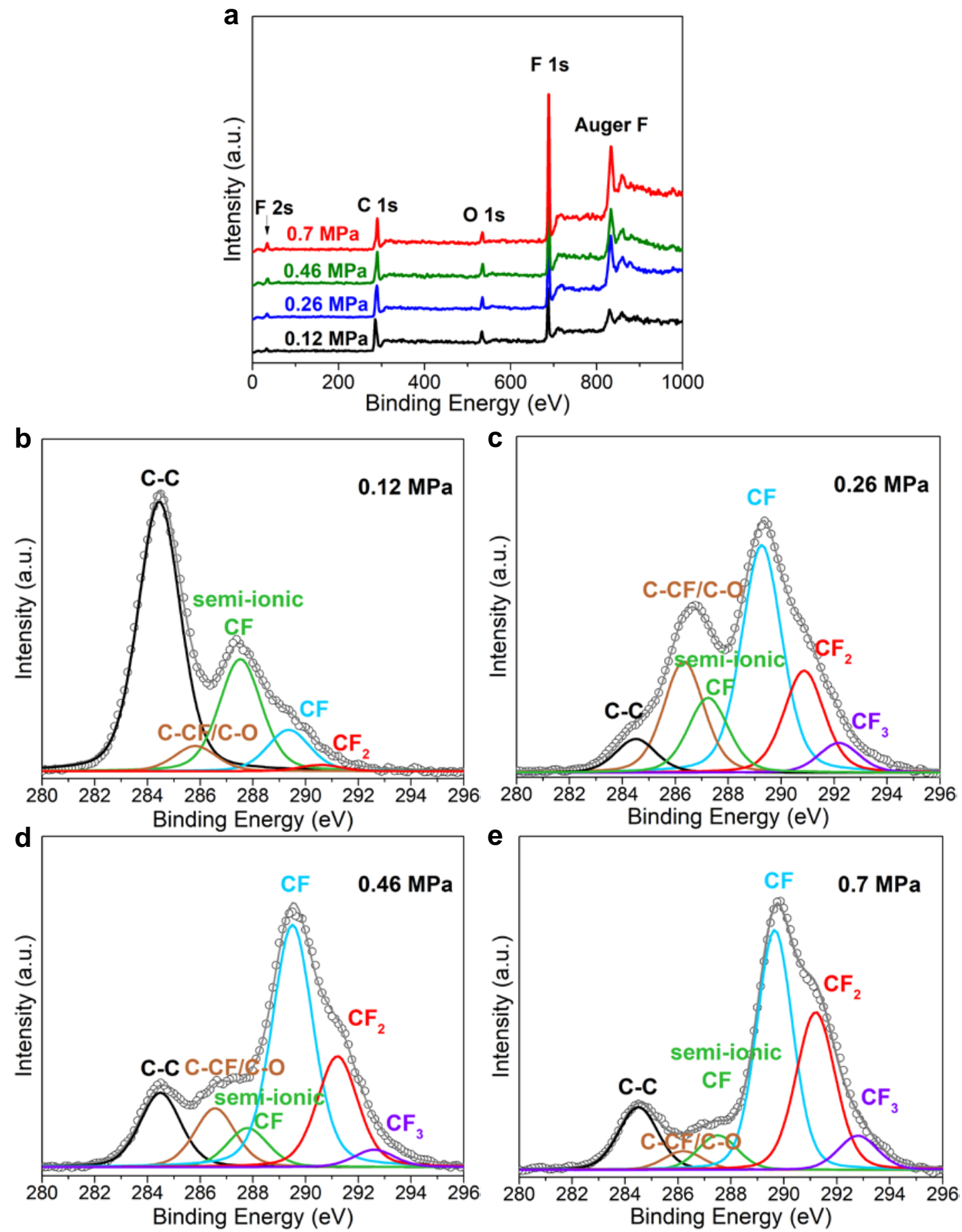

Figure S4. (a) XPS survey spectra and (b)-(e) Fine-scanned C 1s XPS spectra of the FG samples obtained at different fluorination pressures. The dots are measured data and the solid lines are fitted curves. 

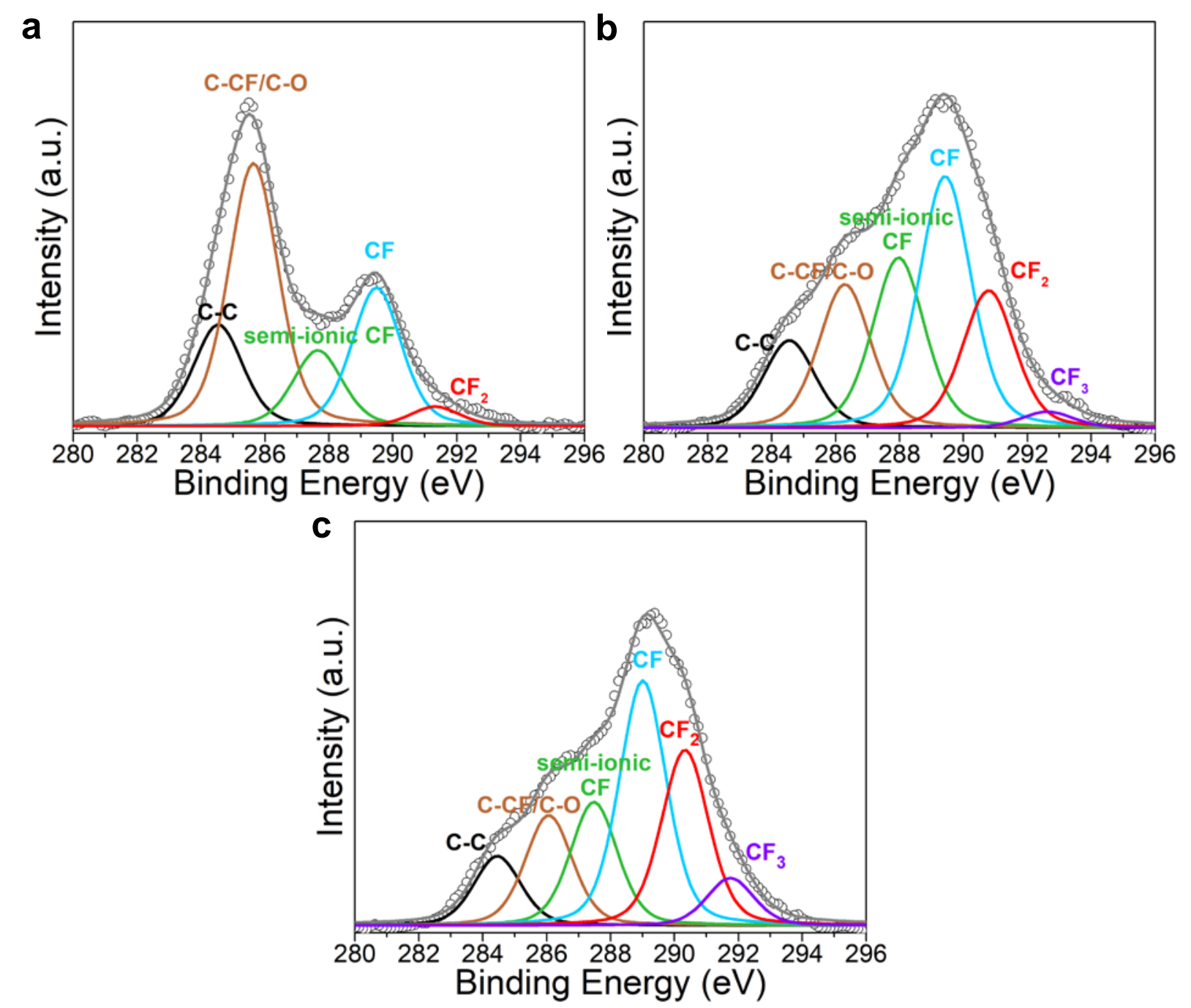

Figure S5. (a)-(c) Fine-scanned C 1s XPS spectra of the F-C60 samples obtained at different fluorination pressures. The dots are measured data and the solid lines are fitted curves. 

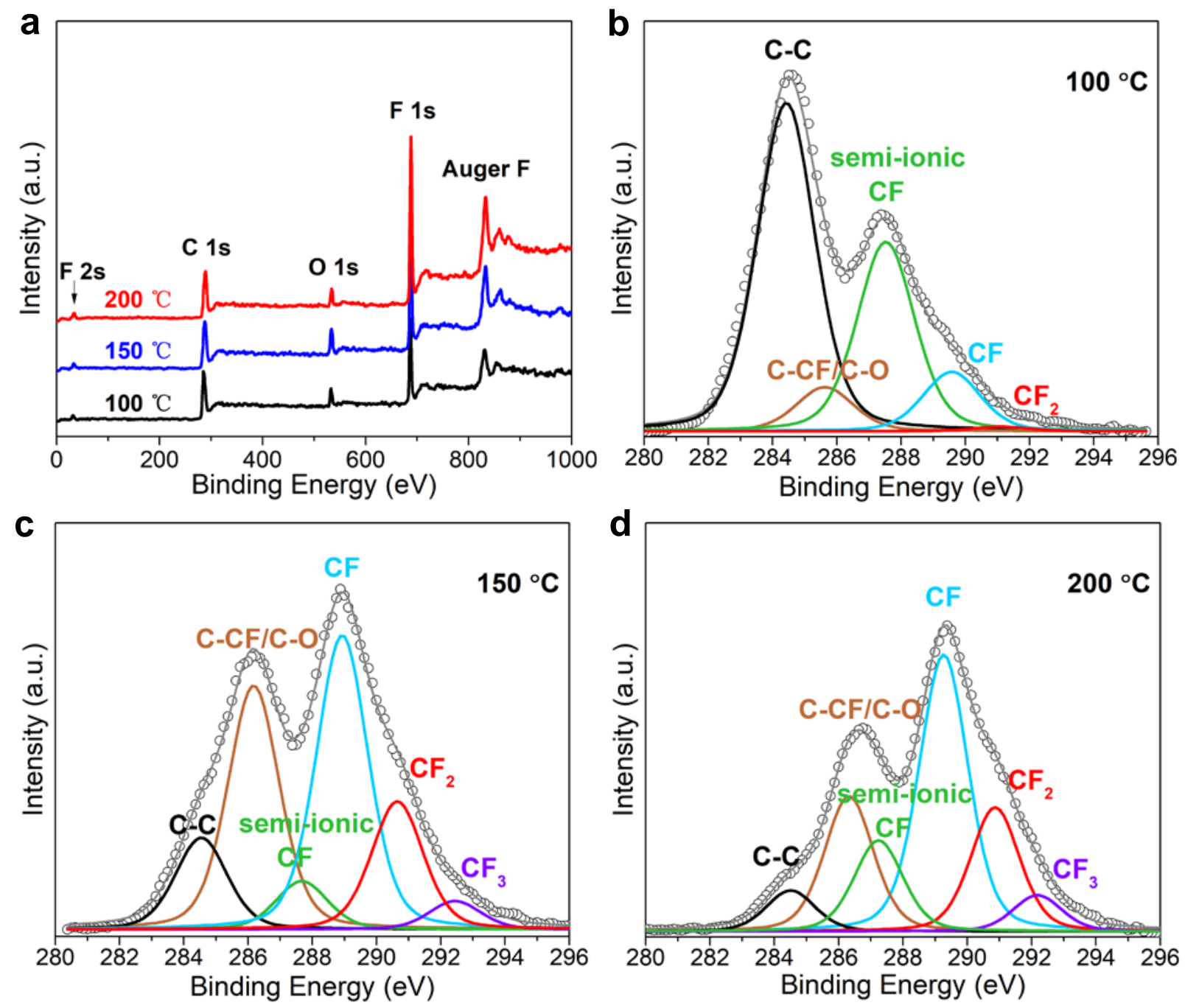

Figure S6. (a) XPS survey spectra and (b)-(d) Fine-scanned C 1s XPS spectra of the FG samples obtianed at different fluorination temperatures. The dots are measured data and the solid lines are fitted curves. 

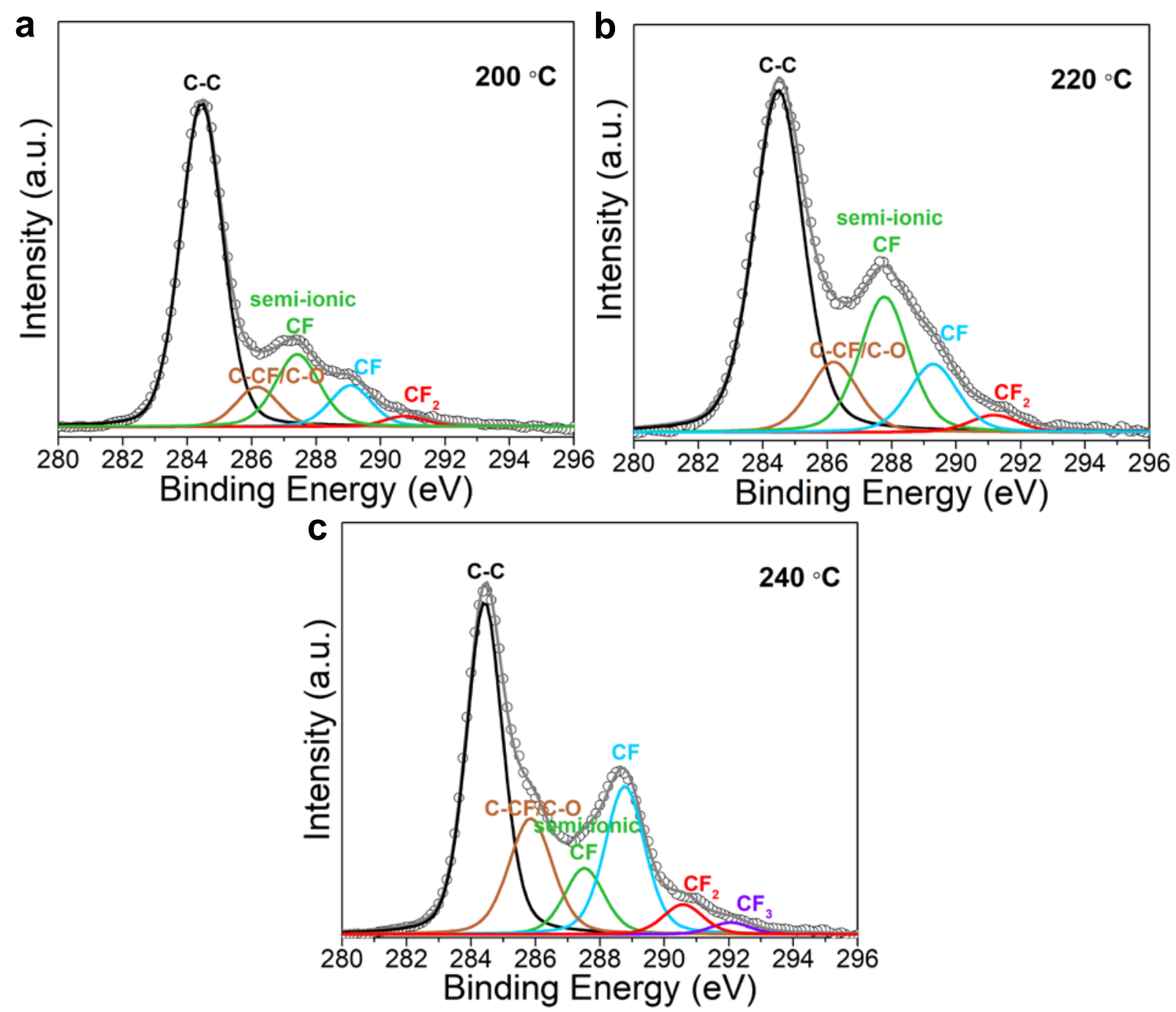

Figure S7. (a)-(c) Fine-scanned C 1s XPS spectra of the F-SWCNTs samples obtained at different fluorination pressures. The dots are measured data and the solid lines are fitted curves. 


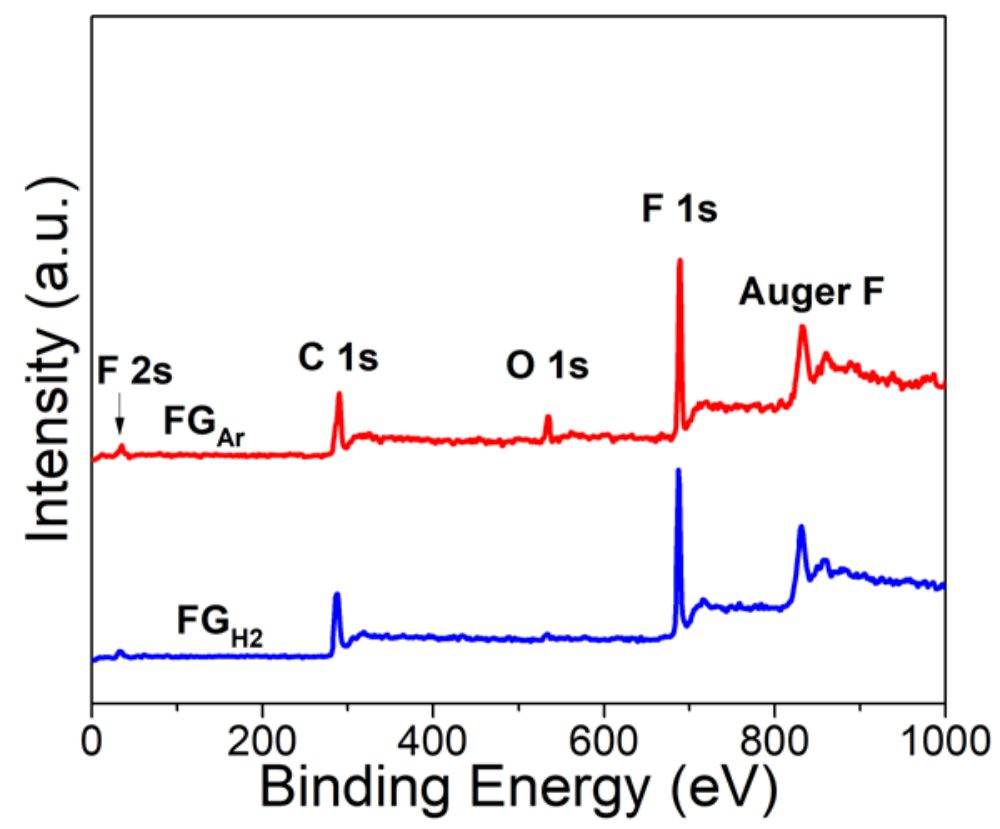

Figure S8. XPS spectra over a wide range of binding energies of FGAr and FGH2. 


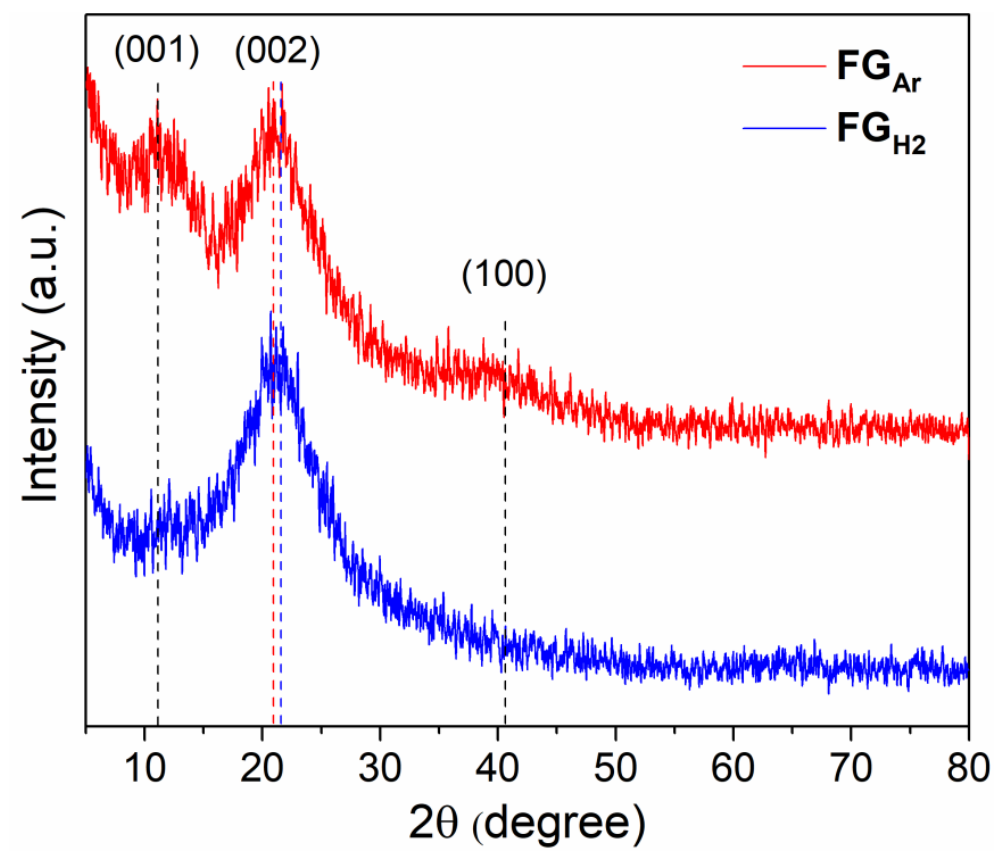

Figure S9. X-ray diffraction spectra of FGAr and FGH2. 


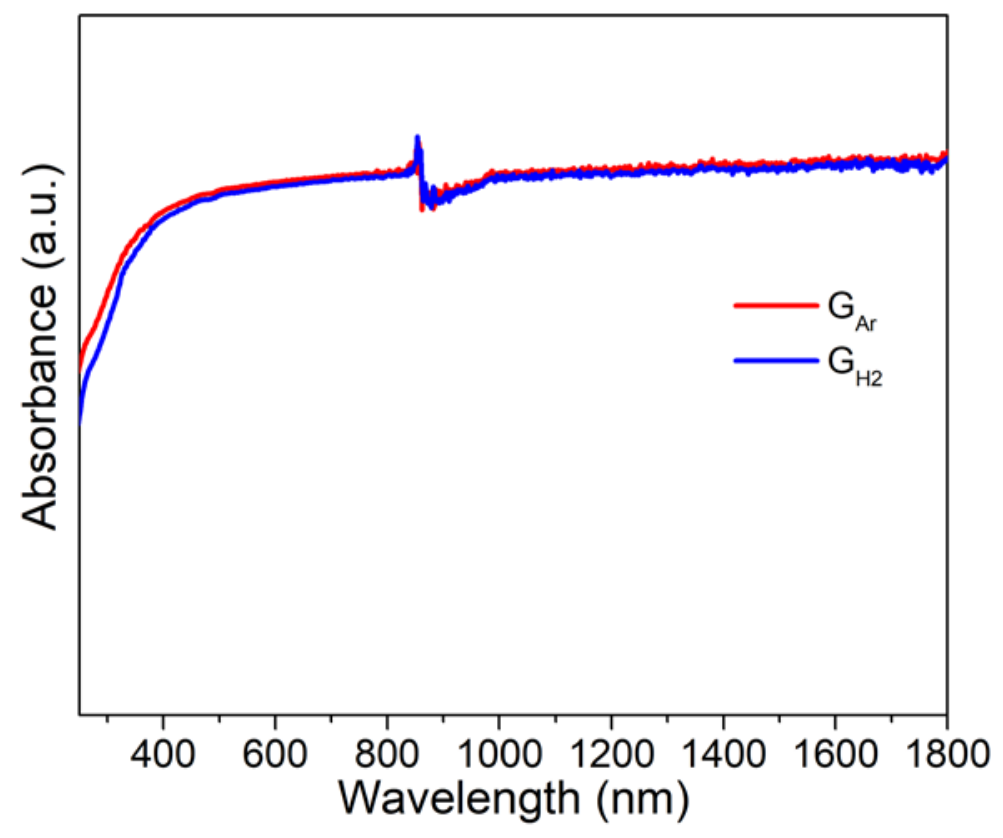

Figure S10. UV-vis absorption spectra of $\mathrm{G}_{\mathrm{Ar}}$ and $\mathrm{GH2}$. It is found that no measurable bandgap of precursors was detected. 

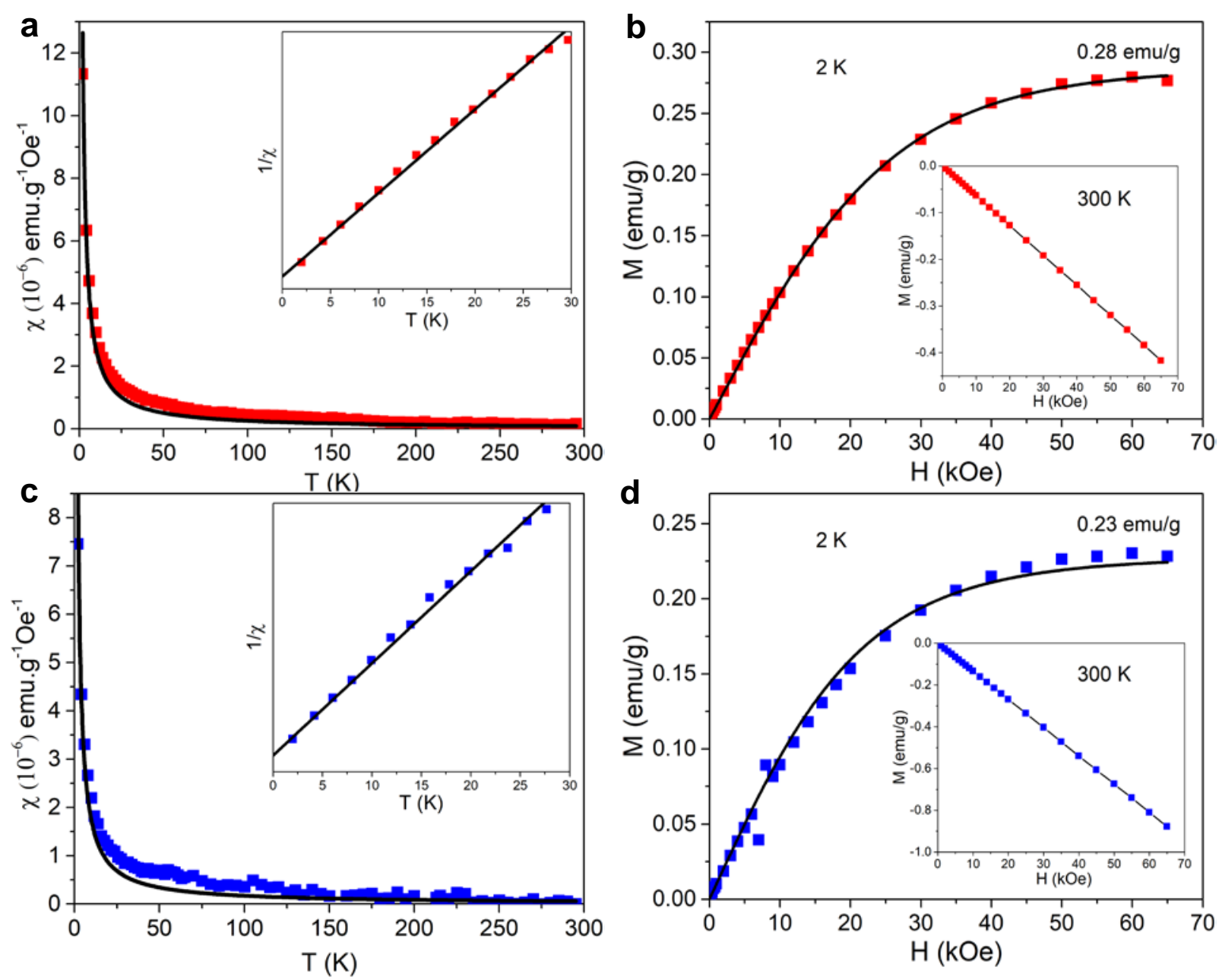

Figure S11. Typical $\chi$ - $T$ curve of (a) $\mathrm{G}_{\mathrm{Ar}}$ and (c) $\mathrm{G}_{\mathrm{H} 2}$ in the applied field $H=1$ kOe. Inset is the corresponding $1 / \chi-T$ curve from 2 to $30 \mathrm{~K}$. $2 \mathrm{~K} \mathrm{M-H}$ curves of (b) $\mathrm{G}_{\mathrm{Ar}}$ and (d) GH2. Inset is $300 \mathrm{~K}$ $M-H$ curves. Symbols are the measurements and solid lines are fitted to Brillouin function. Both of the two precursors show purely Curie-like paramagnetism. The saturation magnetization $M_{\mathrm{s}}$ is 0.28 and $0.23 \mathrm{emu} / \mathrm{g}$ at $2 \mathrm{~K}$, respectively. 

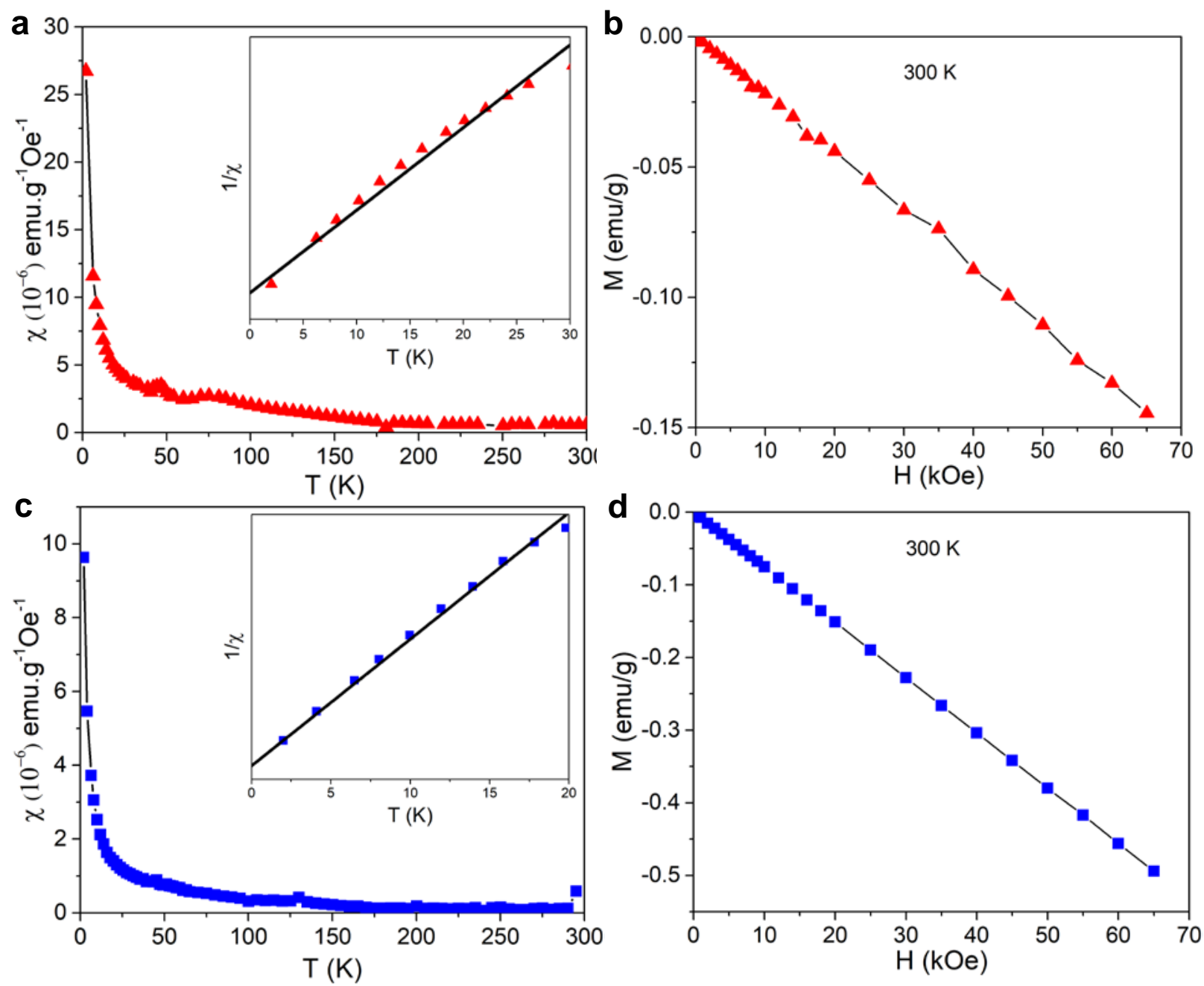

Figure S12. Typical $\chi$ - $T$ curve of (a) FGAr and (c) FGH2 in the applied field $H=1$ kOe. Inset is the corresponding 1/ $\chi$ - $T$ curve from 2 to $20 \mathrm{~K}$. $300 \mathrm{~K} \mathrm{M}-\mathrm{H}$ curves of (b) $\mathrm{FG}_{\mathrm{Ar}}$ and (d) FGн2. The FGн2 shows purely Curie-like paramagnetism. The Curie-like paramagnetism is dominant for $\mathrm{FG}_{\mathrm{Ar}}$, although there is a peak at $50 \mathrm{~K}$. 
Table S1. Ratios of different groups in carbon bonding for FG, F-SWCANTs and F-C60 fluorinated at $200{ }^{\circ} \mathrm{C}$ for $28 \mathrm{~h}$. 'ND' denotes 'not found'.

\begin{tabular}{cccc}
\hline Ratio (\%) & FG & F-SWCNTs & F-C60 \\
\hline C-C & 11.60 & 45.05 & 9.00 \\
C-CF/C-O & 2.60 & 10.07 & 14.30 \\
semi-ionic CF & 5.63 & 12.71 & 31.74 \\
CF & 48.63 & 24.07 & 22.78 \\
CF2 & 26.99 & 6.54 & 6.15 \\
\hline
\end{tabular}


Table S2. Ratios of different groups in carbon bonding for $\mathrm{FG}_{\mathrm{H} 2}$ and FGAr. 'ND' denotes 'not

\begin{tabular}{ccc} 
found’. & & \\
\hline Ratio (\%) & FGH2 & FG \\
\hline C-C & 2.51 & 15.41 \\
C-CF/C-O & 47.00 & 11.87 \\
semi-ionic CF & ND & 10.28 \\
CF & 42.73 & 38.09 \\
CF2 & 7.76 & 15.76 \\
$\mathrm{CF}_{3}$ & $\mathrm{ND}$ & 8.60 \\
\hline
\end{tabular}


Table S3. The contents of the metal impurity elements of graphene, FGAr and FGн2 samples measured by the ICP spectrometry. The unit is ppm. 'ND' denotes 'not found'.

\begin{tabular}{cccccc}
\hline Sample & Fe & Co & Ni & Cr & Mn \\
\hline GAr & 16 & ND & ND & ND & ND \\
GH2 & ND & ND & ND & ND & ND \\
FGAr & ND & ND & ND & ND & ND \\
FGH2 & ND & ND & ND & ND & ND \\
\hline
\end{tabular}

\section{REFERENCES}

(1) Marcano, D. C.; Kosynkin, D. V.; Berlin, J. M.; Sinitskii, A.; Sun, Z.; Slesarev, A.; Alemany, L. B.; Lu, W.; Tour, J. M. Improved Synthesis of Graphene Oxide. ACS Nano 2010, 4, 48064814.

(2) Feng, Q.; Tang, N. J.; Liu, F. C.; Cao, Q. Q.; Zheng, W. H.; Ren, W. C.; Wan, X. G.; Du, Y. W. Obtaining High Localized Spin Magnetic Moments by Fluorination of Reduced Graphene Oxide. ACS Nano 2013, 7, 6729-6734. 